\title{
Response of the inner radiation belt to the violent Sun-Earth connection events of October-November 2003
}

\author{
M. D. Looper and J. B. Blake \\ The Aerospace Corporation, Los Angeles, California, USA \\ R. A. Mewaldt \\ Physics Department, California Institute of Technology, Pasadena, California, USA \\ Received 14 September 2004; revised 21 October 2004; accepted 1 November 2004; published 11 January 2005.
}

[1] We report observations of the response of the lowaltitude radiation population below $\mathrm{L}=3$ during and after the strong solar energetic particle events and geomagnetic disturbances of late October and early November 2003, and we place this response in the context of observations throughout the 12-year SAMPEX mission. We find that on 29 October 2003, at the approximately $600 \mathrm{~km}$ altitude of SAMPEX, the usual belt of energetic protons (above $19 \mathrm{MeV}$ ) around $\mathrm{L}=2$ almost completely disappeared, recovering only after several months. We also observed the appearance of a new belt of ultrarelativistic (above $10 \mathrm{MeV}$ ) electrons centered around $\mathrm{L}=2$. In the previous twelve years, we have never observed such a large decrease of the energetic protons at low altitude. An injection of very high energy electrons like this has not been seen since February 1994. Citation: Looper, M. D., J. B. Blake, and R. A. Mewaldt (2005), Response of the inner radiation belt to the violent Sun-Earth connection events of October-November 2003, Geophys. Res. Lett., 32, L03S06, doi:10.1029/2004GL021502.

\section{Introduction}

[2] From 28 October 2003 into early November 2003, the Earth was exposed to a sequence of solar energetic particle (SEP) events that included one of the most intense ones ever observed; the geomagnetic response was also powerful, with Dst reaching $-400 \mathrm{nT}$. The region of the Earth's inner radiation belt (inside about $\mathrm{L}=3$ ) is shielded from the effects of most SEP events and geomagnetic disturbances, varying mainly on the 11-year timescale of the solar activity cycle. However, particularly strong disturbances can have effects that reach to very low $\mathrm{L}$ values, with the best-known example being the sudden injection inside $\mathrm{L}=3$ of electrons and protons with energies above $15 \mathrm{MeV}$ on 24 March 1991 [Blake et al., 1992]. Less severe disturbances can inject transient belts of protons with energies up to tens of $\mathrm{MeV}$ into the slot region ( $L=2$ to 3 ) immediately outside the CRAND proton belt, where they can persist until a subsequent geomagnetic storm distorts the local magnetic field so that the protons cease to be adiabatic [Blake et al., 2005].

[3] In this paper we present observations from the Proton/ Electron Telescope (PET) aboard the Solar, Anomalous, and Magnetospheric Particle Explorer (SAMPEX), which was launched into a $600 \mathrm{~km}$ near-polar orbit in July 1992 [Cook et al., 1993]. PET consists of a stack of twelve silicon solid- state wafers 2 to $3 \mathrm{~mm}$ thick, grouped into eight detectors, with up to three pulse-height measurements available per particle. The combination of range and multiple pulseheight information in this $\mathrm{dE} / \mathrm{dx}$ telescope allows us to distinguish protons and electrons with confidence, even in regions where one or the other species dominates.

\section{Protons}

[4] Figure 1 shows daily averages of protons at 19$29 \mathrm{MeV}$ as a function of L (IGRF) for a period of nine months around the time of these SEP events. The trapped protons, peaked around $\mathrm{L}=2$, are visible at the start of the period, and the SEP events are visible as bands stretching down from high L (when SAMPEX was over the polar caps). SAMPEX samples the South Atlantic Anomaly region, where the trapped protons can be observed at low altitude, with roughly 12-hour resolution (as the rotation of the Earth brings these longitudes under the ascending and descending legs of the spacecraft's polar orbit), so PET cannot pinpoint timing of changes with a resolution of better than one-half day. On 29 October 2003 we saw that the low-altitude protons had disappeared almost completely. Looking at later times in Figure 1, it can be seen that it took several months for the protons to recover to their former intensity.

[5] Figure 2 places this event in the context of observations of count rate $v s$. L over the 12-year SAMPEX mission (through June 2004). In order to avoid artifacts due to the finite aperture of PET, the one- to three-month averages plotted here only include protons observed with local pitch angles within 10 degrees of perpendicular to the local magnetic field; in order to average out the effects of spacecraft position and pointing, the averages only include protons with pitch angles that, projected to the highest altitude (lowest B magnitude) reached by SAMPEX on the proton's L shell, would have been within 26 degrees of perpendicular to the field there. These are the same cuts used in our earlier study of hydrogen isotopes in the inner zone [Looper et al., 1996]; the resulting plot of the intensity of the protons mirroring at or near the highest altitude reached by SAMPEX on each field line is nearly free of artifacts caused by the conditions of observation. (There is some dilution of intensity starting in about 2000 in a plot made with these cuts, as the SAMPEX orbit decays and the highest fluxes at the highest altitudes are no longer sampled.) The plot shows the increasing low-altitude energetic proton flux during solar minimum (around 1996) and the decrease toward the following solar maximum, and there is some fine-timescale 


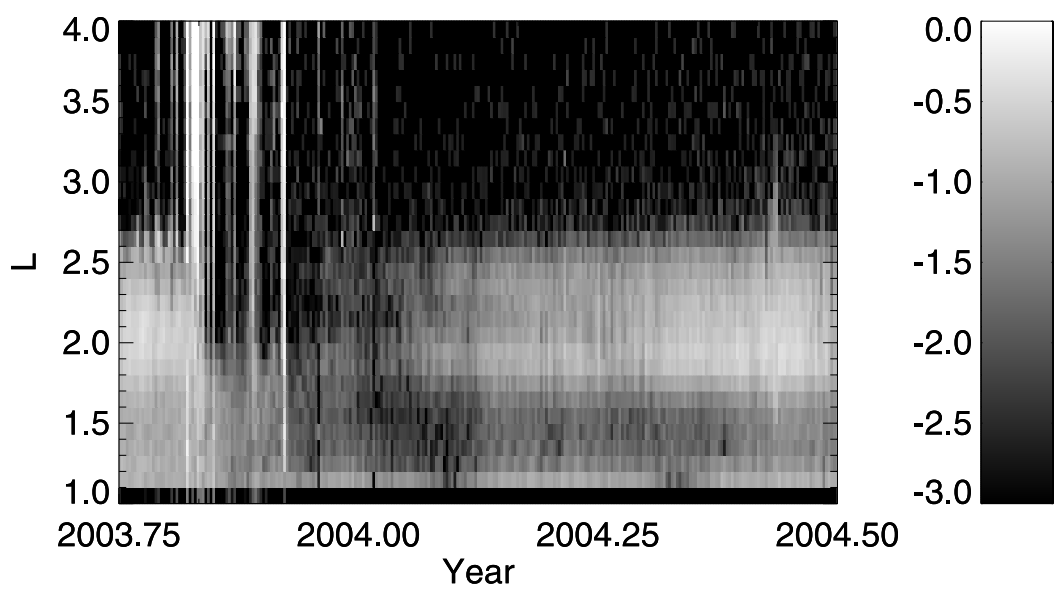

Figure 1. Daily averages of 19-29 MeV proton count rate vs. L and time for nine months around the October/November 2003 events. Color scale is the logarithm (base 10) of the count rate per second in all figures. See color version of this figure in the HTML.

structure during solar maximum, but in the entire mission before October 2003 there are no dropouts of flux as complete or long-lasting as the one observed at that time.

[6] Figure 3 is a plot of proton count rate $v s$. L over the entire SAMPEX mission made with the same cuts as Figure 2, but for protons at a higher energy of 86$120 \mathrm{MeV}$. Note that the peak intensities are at substantially lower L. The trends with solar cycle phase visible in Figure 2 are seen here, though with less fluctuation of the position of the outer boundary at the higher energy, and again the October 2003 dropout is unprecedented in depth and duration. Count rates of protons at other energies up to $500 \mathrm{MeV}$ show similar behavior.

\section{Electrons}

[7] PET can distinguish electrons above about $2 \mathrm{MeV}$ from protons; usually the bulk of electrons at multi-MeV energies are found at higher $\mathrm{L}$ than we are considering here, though geomagnetic activity can cause injections of multi$\mathrm{MeV}$ electrons into the slot region. Such an injection was observed in the PET 2-6 MeV electron channel during the active period of October/November 2003, but this event was not at all exceptional in intensity or duration compared to others observed during the SAMPEX mission.

[8] The highest-energy electrons observed by PET, at 10$20 \mathrm{MeV}$, are by contrast quite rare anywhere in the magnetosphere. The vast majority of the fluence of these particles accumulated over the entire SAMPEX mission has been from the remnants of the electrons injected on 24 March 1991. Figure 4 is a plot like Figure 1, showing daily averages of count rate $v s$. L over nine months in our highest-energy electron channel; the low fluxes of these particles combined with details of the PET response to them result in a plot with much poorer statistics than Figure 1. Nonetheless, ultrarelativistic electrons can be seen among the SEPs as a band reaching down from high $\mathrm{L}$ during the most intense period of activity, around 30 October 2003 (indicated by an arrow); moreover, some months after the period of SEP events, a new belt of ultra-relativistic electrons began to appear at about $\mathrm{L}=2$ and persisted at least until July 2004, when data collection from SAMPEX ceased.

[9] Figure 5 is, like Figures 2 and 3, a homogeneous plot of count rate vs. L over the entire mission, for the $10-$
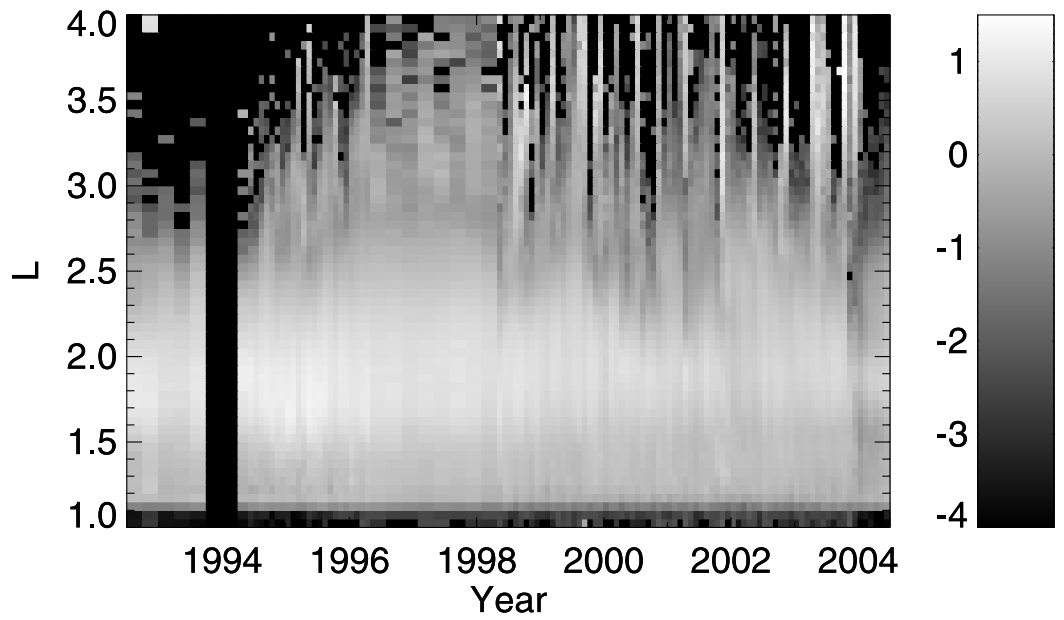

Figure 2. Average count rate of $19-29 \mathrm{MeV}$ protons mirroring near the SAMPEX maximum altitude, vs. L and time for the entire mission. See color version of this figure in the HTML. 


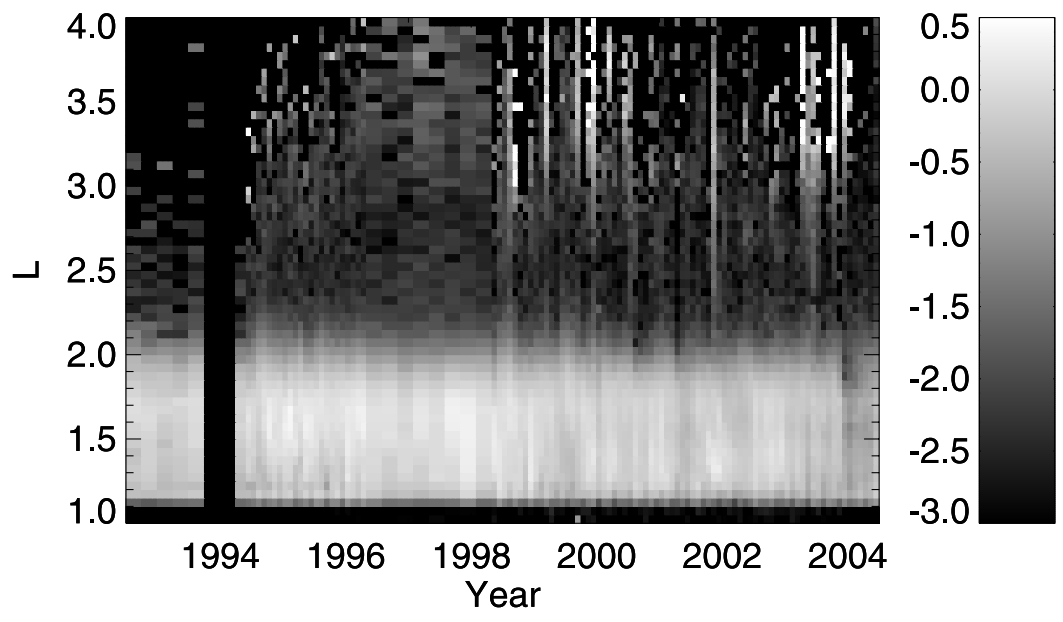

Figure 3. Average count rate of $86-120 \mathrm{MeV}$ protons mirroring near the SAMPEX maximum altitude, vs. L and time for the entire mission. See color version of this figure in the HTML.

$20 \mathrm{MeV}$ electrons. As noted above, the most prominent population of these particles during the mission is the remnants of the March 1991 belt, seen at about L = 2 (saturating the color scale) from mission start. In February 1994, we observed the appearance of a new population around $\mathrm{L}=2.5$; subsequently this merged with the remnants of the March 1991 belt, and these electrons moved inward below $\mathrm{L}=1.5$. However, after 1994 no new ultra-relativistic electron belts were observed until the events of late 2003.

\section{Discussion}

[10] It will be noted that, in contrast to the abrupt dropout of the proton flux, the appearance of the new ultra-relativistic electron belt was delayed by months following the period of intense SEP and geomagnetic activity, and built slowly in intensity. We think that this is a consequence of the low altitude of the SAMPEX orbit, assuming that the injection event had properties similar to those of the March 1991 abrupt injection. When the CRRES satellite, in a nearequatorial orbit, first observed that event [Blake et al., 1992], the pitch angles of the injected particles were strongly peaked in the direction perpendicular to the magnetic field. Over the remainder of the CRRES mission, the equatorial pitch-angle distribution of the particles broadened, and only over time did they spread out along the field lines to reach SAMPEX altitudes in significant numbers. Had SAMPEX been in its low-altitude orbit in March 1991, then, it would not have detected a strong flux of injected particles until months after the injection, much as is seen in Figure 4.

[11] There is perhaps some hint in Figure 4 of a faint trace of particles moving inward from about $\mathrm{L}=2.5$ in midNovember 2003 until the appearance of a new belt becomes clear in January 2004, but it is a hint at best. (The development of the February 1994 ultra-relativistic electron injection shows a similar pattern, but observational artifacts from changes in the SAMPEX pointing algorithm and in the PET operating mode confuse the issue considerably; this is why the period in late 1993 and early 1994 is blanked out in Figures 2, 3, and 5.) Early SAMPEX observations of the March 1991 electrons [Looper et al., 1994] showed that their intensity decayed on a timescale of six months to a year; while only six months passed from the first clear appearance of the late-2003 belt to the end of SAMPEX

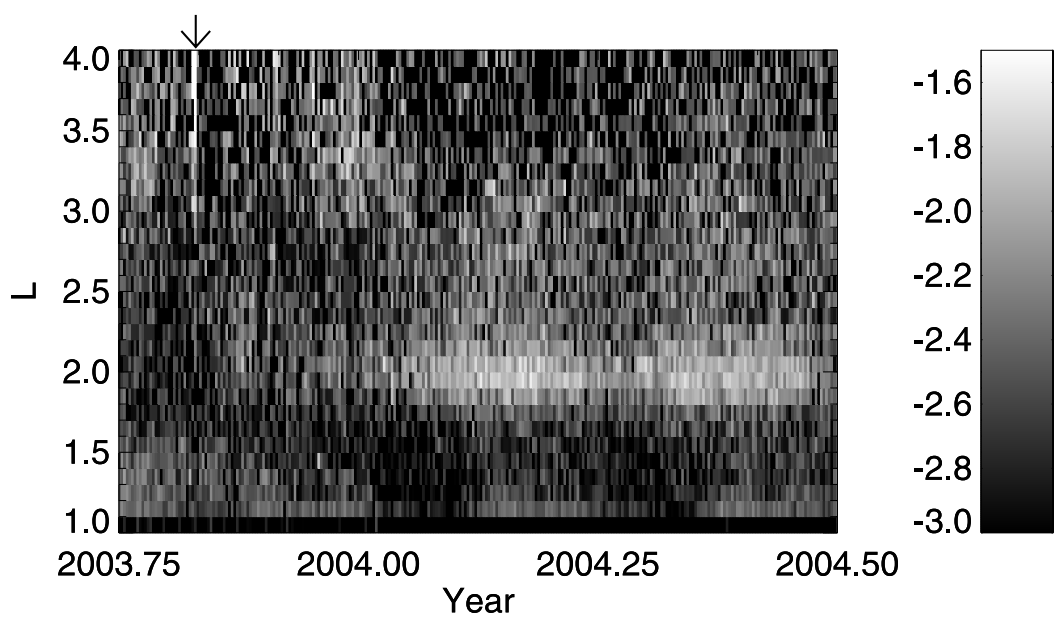

Figure 4. Daily averages of 10-20 MeV electron count rate vs. L and time for nine months around the October/November 2003 events. Arrow indicates 30 October, when the SEP electrons in this energy range were most intense. See color version of this figure in the HTML. 

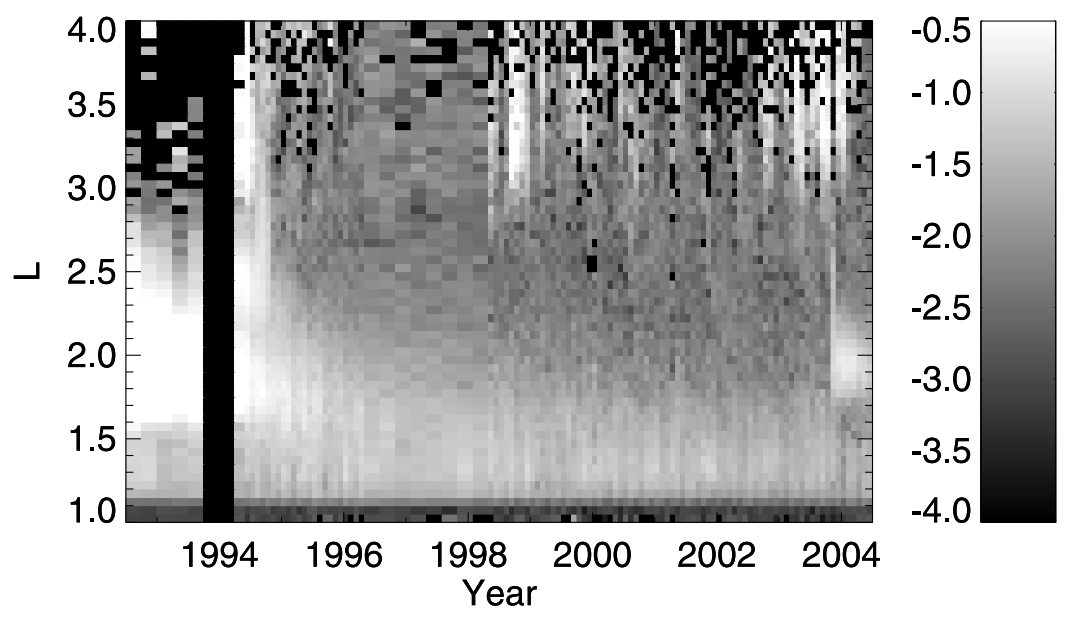

Figure 5. Average count rate of 10-20 MeV electrons mirroring near the SAMPEX maximum altitude, vs. L and time for the entire mission. See color version of this figure in the HTML.

data, the rate of decay of the intensity in this period is consistent with the earlier result.

[12] It was mentioned above that protons with energies up to tens of $\mathrm{MeV}$ are sometimes injected [Hudson et al., 1997; Lorentzen et al., 2002; Blake et al., 2005]. Since the high-L boundary of the steady belt is set for a given proton energy by the limit where the magnetic field at the equator is no longer strong enough to keep the motion adiabatic, it is not surprising that these transient extensions to the belt disappear when new geomagnetic activity depresses the field magnitude and moves that limit inward in L. It is difficult to see how even a strong geomagnetic disturbance could depress the magnetic field enough to disrupt proton gyro-orbits throughout the inner zone and slot, but on 29 October 2003 the flux was observed to drop out at all energies across these regions. Other possibilities include an abrupt, large increase in atmospheric density at SAMPEX altitudes due to the strong radiation input (photons and particles), which would increase the Coulomb drag dramatically, or a sudden increase in plasma wave activity that rapidly scattered protons into the atmosphere. The former mechanism would inherently affect only protons that mirror at low altitudes; the latter mechanism would have to be highly tuned so as to affect only these protons, since otherwise the low-altitude protons would be replenished by pitch-angle scattering of particles that previously mirrored closer to the equator. Deciding what mechanism emptied out the low-altitude proton population requires data from sensors we do not have aboard SAMPEX. A substantial increase in low altitude atmospheric density may be detectible from low-altitude satellite drag observations. Finally, we note that the rate of refilling of the low-altitude inner zone with protons provides an opportunity for comparison with models of the proton source and/or of the scattering of protons to low altitudes.

[13] In summary, the violent Sun-Earth connection events of late 2003 produced effects on the energetic particles in the deep inner magnetosphere that have few or no precedents over the past solar cycle. Detecting such rare and dramatic effects is partly a matter of luck, much as CRRES was in the "right place at the right time" to observe the March 1991 injection; however, placing them in context demonstrates the enormous value of a long-running intra-calibrated data set developed while "lying in wait" for these rare events.

[14] Acknowledgments. This work was supported by contract Z667103 between the University of Maryland and The Aerospace Corporation and by contract Z667101 between the University of Maryland and Caltech. We would like to acknowledge the contributions of the PET team, who developed and built the instrument, and of the personnel at Goddard Space Flight Center who constructed, launched, and operated the SAMPEX spacecraft.

\section{References}

Blake, J. B., M. S. Gussenhoven, E. G. Mullen, and R. W. Fillius (1992), Identification of an unexpected space radiation hazard, IEEE Trans. Nucl. Sci., 39, 1761-1764.

Blake, J. B., P. L. Slocum, J. E. Mazur, M. D. Looper, R. S. Selesnick, and K. Shiokawa (2005), Geoeffectiveness of shocks in populating the radiation belts, in Multiscale Coupling of Sun-Earth Processes, edited by A. T. Y. Lui, Y. Kamide, and G. Consolini, Elsevier, New York, 125-134. Cook, W. R., et al. (1993), PET: A Proton/Electron Telescope for studies of magnetospheric, solar, and galactic particles, IEEE Trans. Geosci. Remote Sens., 31, 565-571.

Hudson, M. K., S. R. Elkington, J. G. Lyon, V. A. Marchenko, I. Roth, M. Temerin, J. B. Blake, M. S. Gussenhoven, and J. R. Wygant (1997), Simulation of radiation belt formation during storm sudden commencements, J. Geophys. Res., 102, 14,087-14,102.

Looper, M. D., J. B. Blake, R. A. Mewaldt, J. R. Cummings, and D. N. Baker (1994), Observations of the remnants of the ultrarelativistic electrons injected by the strong SSC of 24 March 1991, Geophys. Res. Lett., $21,2079-2082$

Looper, M. D., J. B. Blake, J. R. Cummings, and R. A. Mewaldt (1996), SAMPEX observations of energetic hydrogen isotopes in the inner zone, Radiat. Meas., 26, 967-978.

Lorentzen, K. R., J. E. Mazur, M. D. Looper, J. F. Fennell, and J. B. Blake (2002), Multi-satellite observations of $\mathrm{MeV}$ ion injections during storms, J. Geophys. Res., 107(A9), 1231, doi:10.1029/2001JA000276.

J. B. Blake, The Aerospace Corporation, P.O. Box 92957-M2/259, Los Angeles, CA 90009-2957, USA

M. D. Looper, The Aerospace Corporation, P.O. Box 92957-M2/260, Los Angeles, CA 90009-2957, USA. (mark.d.looper@aero.org)

R. A. Mewaldt, Physics Department, 220-47 Downs Laboratory, Caltech, Pasadena, CA 91125, USA. 